\title{
SECTION II:
}

SPECTROGRAPHS AND SPECTROMETERS 


\author{
Dianne L. Harmer \\ Royal Greenwich Observatory \\ Hailsham, East Sussex. BN27 1RP
}

\begin{abstract}
Grism" and compound prism cross-dispersers have been proposed for the new Cassegrain spectrograph of the $2.5 \mathrm{~m}$ telescope at La Palma. The RGO image-tube spectrograph has been fitted with a grism to provide us with experience in designing layouts, operating the equipment and handling the data.
\end{abstract}

Instrument design for large telescopes frequently starts out with the astronomical community being undecided upon whether to choose single purpose systems which may be exceptionally efficient for specialised applications or try to find efficient layouts with multiple options to provide more versatile equipment.

In 1977, during the initial stages of the design of the new Cassegrain spectrograph for the $2.5 \mathrm{~m}$ Isaac Newton Telescope on La Palma, proposals were made to include cross-dispersion facilities for both low- and intermediate-dispersion spectroscopy. By that time it had already been agreed that the system should be capable of operating with a long slit ( 4 arc min), which would therefore allow room in the image format for a few orders to be recorded with modest separations.

The spectrograph is a flat-bed arrangement, having two folded "short Schmidt" cameras (Wynne (1977)) of $23.5 \mathrm{~cm}$ and $50.0 \mathrm{~cm}$ focal length. At RGO, in 1977, Morris made a study of the geometry of the system to obtain optimum throughput; the resulting layout is shown in Fig. 1. Dispersions from $130 \mathrm{~A} \mathrm{~mm}^{-1}$ to $7 \mathrm{~A} \mathrm{~mm}^{-1}$ are available in the standard mode, but spectral coverage is incomplete at intermediate dispersions because of limited field size, and at low dispersion overlapping orders prevent full use of the detectable wavelength range.

The two spectrograph cameras are designed for use with interchangeable gratings, working at attitudes which produce a modest range of anamorphic magnification. Substitution of a very high blaze angle echelle grating to obtain the multi-order format would therefore not be acceptable, so a variety of low ruling frequency gratings have been

109

C. M. Humphries (ed.), Instrumentation for Astronomy with Large Optical Telescopes, 109-116. Copyright $\odot 1982$ by D. Reidel Publishing Company. 


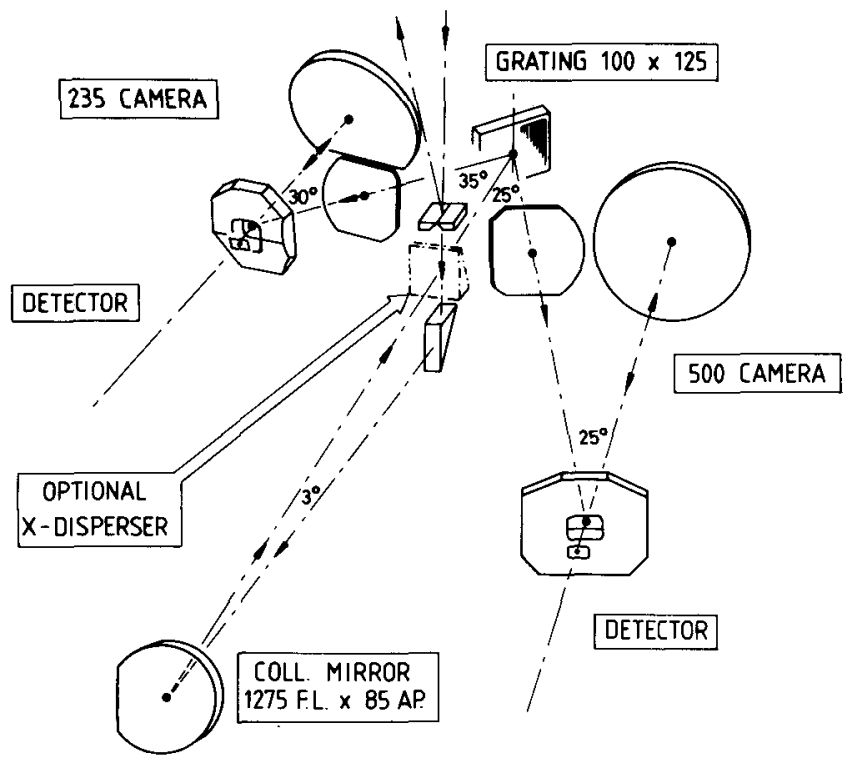

Figure 1. Arrangement of optical components in the Cassegrain spectrograph for the $2.5 \mathrm{~m}$ INT at La Palma. The instrument is mounted on the acquisition and guiding unit at $f / 15$ focus. Camera focal lengths are $23.5 \mathrm{~cm}$ and $50 \mathrm{~cm}$ respectively, and the design is based on an $85 \mathrm{~cm}$ collimator beam diameter.

examined for free spectral range and efficiency characteristics when used in higher orders than usual. Estimates of efficiencies supplied by the grating manufacturers looked encouraging, so prime dispersers were selected from ordinary reflection gratings. Both grating and prism cross-dispersion were proposed, but since the mechanical arrangement of the spectrograph is fixed, the former had to be a transmission grating-prism ("grism"), whilst the latter had to produce dispersion without deviation. In each case, the effect of introducing the cross dispersion had to be controlled to a level where performance of the imaging optics remained satisfactory. Other criteria affecting the combinations chosen relate to the type of detector used, its associated memory size and dimensions of individual elements. In the case of photographic plates or image tubes the entire image format can be recorded at full resolution over the whole field. The INT spectrograph will be fitted with the UCL Image Photon Counting System (Boksenberg (1972)) so the external memory size and element sizes will govern the amount of recordable spectrum.

At the time the scheme was proposed, the IPCS external memory could accommodate $192 \mathrm{~K}$ pixels, and several possible layouts were drawn up for grism cross-dispersion including 35-70 $\mathrm{A} \mathrm{mm}^{-1} 3500-7000 \mathrm{~A}$ in 5 
orders and 8-11 $\mathrm{A} \mathrm{mm}^{-1} 3600 \mathrm{~A}-5200 \mathrm{~A}$ in 7 orders, as examples of what might be achieved with maximum resolution and minimum wavelength overlap. Neither of these formats fills the available image areas of $30 \mathrm{~mm}$ $x 8 \mathrm{~mm}$ and $30 \mathrm{~mm} \times 16 \mathrm{~mm}$. It may now be possible for the IPCS to be upgraded to $512 \mathrm{~K}$ pixels (as it is at the Anglo-Australian Telescope), allowing more orders to be recorded at larger separation, and permitting wider spectral coverage at the higher dispersions. Consequently the final choice of combinations of prime and cross-dispersers has been deferred, pending this decision.

The other proposal, a compound prism producing dispersion without deviation, seemed suitable only for the low dispersion option as an alternative to an order-sorting filter which would remove overlapping wavelengths altogether. Mounting requirements constrain the prism thickness, as does the need to maintain image quality in the camera; an order-centre separation equivalent to 20 arc sec can be achieved between first and second orders of the prime disperser (8000 A-4000 A) using a prism of UBK $7\left(12.6^{\circ}\right)$ and LF5 $\left(11.1^{\circ}\right)$. In the grism crossdispersion formats, spectra are essentially straight, but spectrum curvature is significant in the prism system. The deviation is $1.8 \mathrm{~mm}$ over a $30 \mathrm{~mm}$ field from $3000 \mathrm{~A}-7000 \mathrm{~A}$, and adds to the vital need for versatile data reduction software.

Agreement having been reached on the principles of these schemes, an experiment was devised to introduce a cross-dispersing grism into the RGO image tube spectrograph, comparing the predicted performance with results of laboratory and astronomical observations.

Palmer and Milsom (1972) have described the spectrograph, in which a Cassegrain collimator feeds a reflection grating, and the imaging system (Wynne (1971)) is an $\mathrm{f} / 1.4$ semi-solid Maksutov-Cassegrain camera. The form of the collimator and layout of the instrument lead to problems in pupil imagery if any significant field along the slit is required (Harmer (1974)); a field lens has now been added below the slit to allow slit lengths up to $2 \mathrm{~cm}$ to be used. Originally, the system was designed only for on-axis work. It is perhaps a happy accident, therefore, that the camera has a circular secondary mirror and can thus be used with a sizeable field across the usual direction of dispersion. The camera is designed to have a $20 \mathrm{~mm}$ diameter field.

Fig. 2 shows the grism in position. Optical tolerance of the camera unit to the presence of the grism, and mechanical constraints on prism thickness have put practical limits on the working field size of about $13 \mathrm{~mm}$ in the direction of cross-dispersion. On the basis of likely grating efficiencies and resulting spectrum formats, standard gratings were selected from the Bausch and Lomb catalogue:-

Reflection grating $35-53-17-860,150 \mathrm{~g} \mathrm{~mm}^{-1}, \lambda \mathrm{b} 4.0 \mu \mathrm{m}$

Transmission grating 35-54-15-760, $150 \mathrm{~g} \mathrm{~mm}^{-1}, \lambda \mathrm{b} 5750 \mathrm{~A}$, replicated on the hypotenuse of a $9.25^{\circ}$ BK7 prism.

The prime disperser was chosen to cover $3500 \mathrm{~A}-7500 \mathrm{~A}$ in 5 orders, with dispersions $40-80 \mathrm{~A} \mathrm{~mm}^{-1}$. Sufficient cross dispersion was 


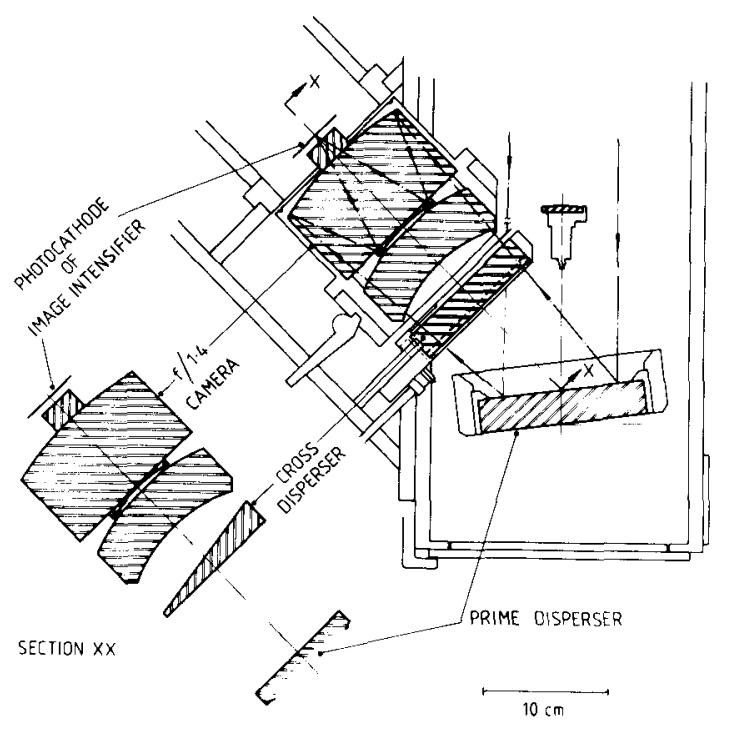

Figure 2. The position of the cross-disperser in the RGo image tube spectrograph is shown here, with the prime disperser at its working attitude, indicating the modest change in beam diameter due to this grating. Obstruction of the down-coming collimated beam can be alleviated by lifting the entire camera unit; the engineering was not done during the testing phase.

required to allow room for a comparison arc between orders, as image tube and photographic plate would be used for recording the spectrum. (The problem of inter-order separation still exists for the low-1ightlevel digital detectors because of the need to record a simultaneous sky spectrum). Harmer (1977) has computed the optical performance of the system and shown that, whilst some image degradation is to be expected, the resolution is still within $30 \mu \mathrm{m}$ FWHM achieved by the EMI image intensifier used as the detector. It is worth digressing briefly at this stage to note a request received from Penston, then at the AAT, to examine performance of grism cross-dispersion in the Wynne (1972) f/1.6 camera of the RGO spectrograph (Palmer and Gietzen (1972)). Harmer's results (1977) showed that this system, too, would be tolerant to the introduction of a modest grism.

Littrow blaze wavelengths of the gratings purchased differed slightly from the catalogue values, being $3.68 \mu \mathrm{m}$ and $5850 \mathrm{~A}$ for the prime and cross-dispersers respectively. The spectrum format achieved by centering the pattern on the effective blaze wavelength is shown in Fig. 3 and the principal lines marked are identified in Table 1. The wavelength range is contained in the 5 th to 9 th orders of the prime disperser; in the ultraviolet, the second order from the grism appears and is stronger than the first order because of the 


\begin{tabular}{|c|c|}
\hline $\begin{array}{l}\text { 1. } 7320 / 30 \mathrm{~A} \text { [OII] } \\
\text { 2. } 6717 / 31 \mathrm{~A} \text { [SII] } \\
\text { 3. } 6548 / 63 / 84 \mathrm{~A}[\mathrm{NII}], \mathrm{H}_{\alpha}, \text { [NII] } \\
\text { 4. } 5876 \mathrm{~A} \mathrm{He} \text { I } \\
\text { 5. } 4959 / 5007 \mathrm{~A}[\mathrm{OIII}]\end{array}$ & $\begin{aligned} 6 . & 4861 \mathrm{~A} \mathrm{HB} \\
7 . & 4471 \mathrm{~A} \mathrm{HeI} \\
8 . & 4340 / 63 \mathrm{~A} \mathrm{H \gamma}, \text { [OIII] } \\
9 . & 4101 \mathrm{~A} \mathrm{H} \delta \\
10 . & 3969 \mathrm{~A} \mathrm{HE} \\
11 . & 3727 / 9 \mathrm{~A} \mathrm{[OII]}\end{aligned}$ \\
\hline
\end{tabular}

Table 1. Identification of principal emission lines attainable with $\mathrm{S} 20$ response, shown in Figure 3 .

transmission grating blaze characteristics. This is not ideal, and arises here from the very limited choice of near-suitable catalogue gratings. With $\mathrm{S} 20$ response, the effect is not serious because of the small amount of extra cross-dispersion provided by the prism. Lowne has kindly measured the efficiencies of the gratings, and his results are shown in Fig. 4. Although the prime disperser is an ordinary blazed grating used in fairly high orders, it is encouraging to note that the peak efficiencies are comparable with those expected from echelles.

Laboratory experiments carried out on the spectrograph during the summer of 1978 included verification of the expected spectrum format, 2-D focus and tilt adjustment, examination of the ripple function

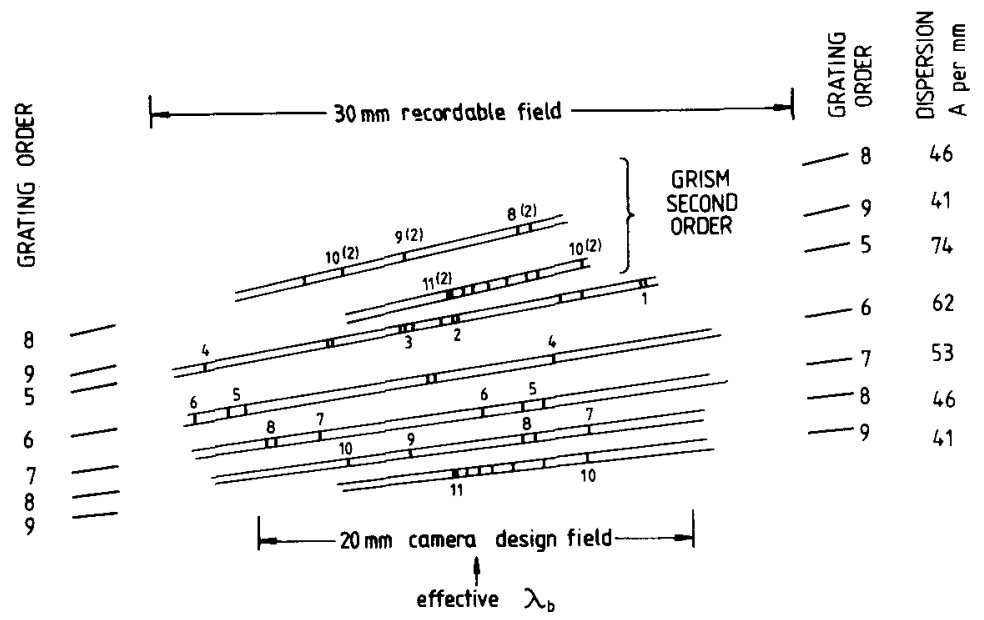

Figure 3. The cross-dispersed spectrum format and positions of principal emission lines attainable with an S20 photocathode. Line identifications are in Table 1 and lines which also appear in the grism second order are designated $\mathrm{n}$ (2). The spectrum height shown corresponds to $300 \mu \mathrm{m}$ at the detector (45 arc sec on the sky at the Cassegrain focus of the RGO 36-inch telescope), and allows arc spectra to be recorded between the orders. 


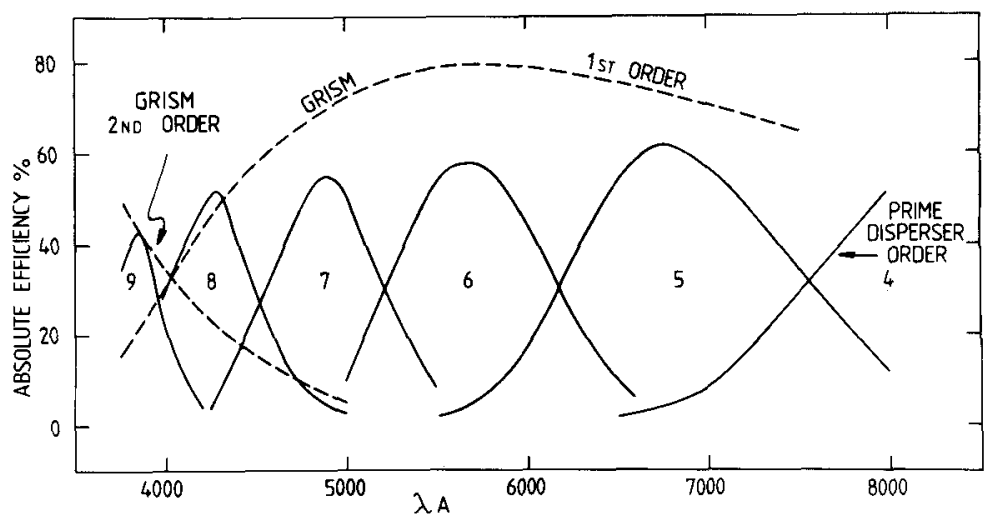

Figure 4. Efficiencies of the prime disperser and transmission grating as measured in the laboratory. These measurements also show how the fairly long blaze wavelength of the transmission grating makes the second order array from order 9 of the prime disperser more prominent than the first order.

using white light spectra and tests of an external photometric calibration system. The spectrograph is fitted with an EMI image intensifier with phosphor output, the image being optically relayed to a IIIaJ photographic plate; emulsion characteristics alone have been used to determine the calibration curve.

Eventually, working equipment and clear skies coincided with an allocation of 36-inch telescope time. During November 1979 and January 1980 Worswick and I obtained observations of the spectrophotometric standard stars HR 718 and HR $1544\left(\pi^{2}\right.$ Ori) using a 5 arc sec slit width and broadening the spectra to $300 \mu m$ (giving room for comparison arcs) or $900 \mu m$ (leaving orders just separated in the ultraviolet). Exposure times were too short to obtain uniform spectra using telescope trail, so the faster electronic trail was used in preference to filters whose neutrality might be questionable. Telescope trail was used with filters only to check illumination with the spectrograph for long-slit observations.

The plates were scanned on a PDS microdensitometer and the data reduced using a suite of computer programs developed at RGO by Burgess, Hartley and Pike based on work originating from Kitt Peak National Observatory. Intensity-calibrated tracings are produced order by order, internally corrected for line slopes.

The standard star observations have been used to derive the relative spectral response for the spectrograph/detector combination and a more detailed account of procedure will appear in a text describing astrophysical results. Fig. 5(a) shows the relative efficiencies 
of the orders derived from two plates of HR 1544 taken at the same air mass ( 1.6 air masses) and with exposures differing by a factor of two so that intensities were obtained from different regions of the photographic characteristic curve. Agreement between the two plates is good, with an r.m.s. scatter in the points of about 5\%; accuracy of photometric photometry used is about $2 \%$ so the scatter is predomi-

a.
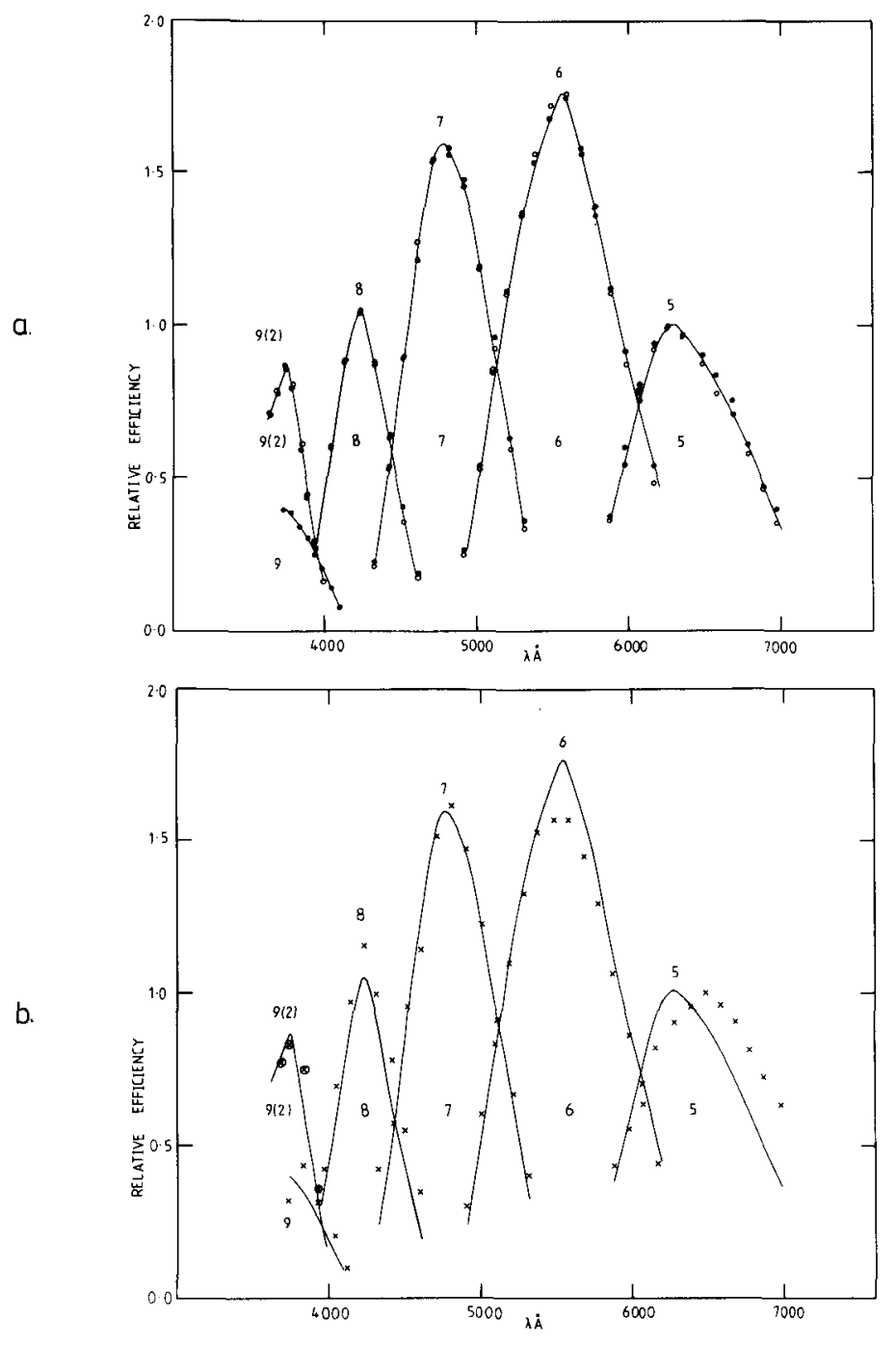

Figure 5(a). Relative efficiency of orders within the cross-dispersed spectrum format, derived from spectra of HR 1544. Filled circles represent measures from a two-minute exposure, open circles from a one-minute exposure. (b) Comparison of predicted (points) and measured (continuous curves) performance of the spectrograph. 
nantly introduced by accuracy of the photographic photometry. A comparison has been made of the performance achieved by the system on the telescope with a "theoretical" efficiency derived from laboratory performance of the gratings and a "typical" S20 sensitivity curve. The points obtained are plotted on the measured curves and shown in Fig. 5(b). Disagreement in the red tail of order 5 is due to a steeper red cut-off in this photocathode sensitivity compared with a "typical" $\$ 20$.

These experiments have shown that it is feasible to use a standard configuration spectrograph with a grism cross-disperser, provided both optical and mechanical parameters are kept within reasonable limits. The system has been used to obtain spectra across an ionisation front in the Orion nebula, showing spatially resolved structure on a scale of the order of $10 \mathrm{arc}$ sec in which line intensity ratios change. These data are being reduced through the computer programs described earlier, but, experiments are also in progress to digitise the data as though it had been obtained on a detector such as the IPCS and attempt to handle it within STARLINK software in readiness for the data emerging from the INT Cassegrain spectrograph.

If this spectrum format is to be transferred into the realms of silicon detectors there will be other problems to overcome. The extended red response already available and the potential lowering of the short wavelength cut-off will make it less attractive to use the grism solution because of overlapping orders. Questions of acceptable order separations and angular sizes of objects will undoubtedly affect the use of prisms alone, as will any changes in mechanical operation if the system is also required to function in a long-slit mode. Experiments on the compound prism proposal have not been carried out, but it is evident that the major problems here will be in extracting and handing the data. Flat field calibration which spans this very large wavelength range $(3500 \mathrm{~A}-1 \mu \mathrm{m})$ presents a significant problem as we11, and it remains to be seen whether the versatile or special instrument becomes the solution to astronomical projects in the future.

\section{REFERENCES}

Boksenberg, A. 1972, Proc. ESO/CERN Conference on Auxiliary Instrumentation for Large Telescopes, ESO, Geneva. p. 295

Harmer, C.F.W. 1974, Mon. Not. R. astr. Soc., 167, p. 311

Harmer, C.F.W. 1977, Private communication

Palmer, D.R. and Milsom, A.S. 1972, Adv. in Electronics and Electron Physics, 33B, p. 769

Palmer, D.R. and Gietzen, J.W. 1972, Proc. ESO/CERN Conference on Auxiliary Instrumentation for Large Telescopes, ESO, Geneva, p. 111

Wynne, C.G. 1971, Mon. Not. R. astr. Soc., 153, p. 261

Wynne, C.G. 1972, Mon. Not. R. astr. Soc., 157, p. 403

Wynne, C.G. 1977, Mon. Not. R. astr. Soc., 180, p. 485 